\title{
Indicadores de desempeño para empresas del sector logístico: Un enfoque desde el transporte de carga terrestre
}

\author{
Performance indicators for logistics enterprises: A land freight transport scope \\ Martín Darío Arango Serna $^{1 *} \quad$ Silvana Ruiz Moreno ${ }^{1} \quad$ Luisa Fernanda Ortiz Vásquez ${ }^{1}$ \\ Julián Andrés Zapata Cortes ${ }^{2}$
}

Recibido 24 de agosto de 2016, aceptado 21 de octubre de 2016

Received: August 24, 2016 Accepted: October 21, 2016

\begin{abstract}
RESUMEN
El presente artículo propone un nuevo marco metodológico para el desarrollo de indicadores que permitan medir el desempeño de las actividades clave para cuatro áreas decisionales identificadas en las empresas de transporte de carga terrestre, siendo estas: gerencia, soporte administrativo, operaciones -logística- y tecnología de procesos. La propuesta teórica se basa en un análisis detallado de literatura científica, de este análisis es posible identificar las actividades clave en cada área decisional de las empresas, al igual que los recursos que se ven afectados por variables internas o externas a la operación y que impactan el desempeño empresarial. Como resultado se propone un marco de indicadores para la medición del desempeño de las empresas de transporte de carga que incluye la definición de cada indicador y cómo calcularlo. Finalmente, se concluye que es posible obtener un conjunto de indicadores que se basen en las necesidades identificadas en literatura científica sin obviar las particularidades de las empresas de transporte de carga, lo que asegura la funcionalidad y el éxito de la implementación de estos indicadores.
\end{abstract}

Palabras clave: Indicadores de desempeño, actividades logísticas, transporte terrestre de carga, Logística.

\begin{abstract}
This paper proposes a new methodological framework for the indicators development that allows measuringe the performance of the key activities on the four decisional areas identified on the land freight transport companies: Management, Administrative support, Operations - Logistics and Process technology. The theoretical proposal is based on a detailed analysis of scientific literature, with this analysis is possible to identify the key activities in each decisional area and the resources that are affected by internal or external variables and impact business performance. As a result, a framework of indicators for measuring the performance of freight companies is proposed, including the definition of each indicator and how to calculate it. Finally, it is concluded that it is possible to obtain a set of signs based on needs identified in scientific literature without forgetting the peculiarities of freight transport companies, which ensures functionality and successful implementation of these indicators.
\end{abstract}

Keywords: Performance indicators, logistics activities, land freight transport, logistics.

\footnotetext{
1 Departamento de Ingeniería de la Organización. Universidad Nacional de Colombia. 050041216. Medellín, Colombia. E-mail:mdarango@unal.edu.co; siruizmo@unal.edu.co; lufortizva@unal.edu.co; jazapat1@unal.edu.co

2 Institución Universitaria CEIPA. Sabaneta, Colombia. E-mail: julian.zapata@ ceipa.edu.co

* Autor de correspondencia.
} 


\section{INTRODUCCIÓN}

El uso de indicadores en las empresas permite obtener información necesaria sobre el estado real de las áreas decisionales que afectan el desempeño de una compañía. Las variables externas y los requerimientos del mercado que son cada vez más exigentes debido a factores como la globalización, la competencia, los cambios en la demanda, etc., hacen que cuantificar el impacto y la evolución de los factores involucrados sea una actividad importante para las empresas.

Un indicador es "una representación cuantitativa, verificable, en la que se registra, procesa y presenta la información necesaria para medir el avance o retroceso de un determinado objetivo" [1]. En [2] se indica que el uso de indicadores y la gestión del desempeño dentro de una empresa del sector logístico, consiste en establecer un conjunto de objetivos y estrategias, definir métricas, cuantificar, analizar, evaluar y actuar, adicional a esto en [3] señalan que los indicadores deben permitir la medición cuantitativa de actividades para así tener la posibilidad de analizar qué aspectos deben abordarse para mejorar algún proceso. Teniendo en cuenta lo anterior, se pueden proponer indicadores que permitan medir el funcionamiento de las empresas del transporte de carga y con estos determinar las falencias que se están presentando y poder tomar decisiones asertivas para la empresa con el fin de mejorar su competitividad.

El seguimiento, medición y control de la operación de una empresa, permite identificar las áreas problemáticas y emprender acciones correctivas para mejorar el desempeño general de la compañía. Los indicadores pueden contribuir a la obtención de ventajas competitivas, para ello deben seleccionarse qué clase de indicadores quieren implementarse y con qué fin [4].

Según [5] en la actualidad no hay un conjunto de reglas sobre las que se puede medir el desempeño de la cadena logística y las métricas presentadas por documentos gubernamentales se enfocan en el desarrollo de indicadores desde el Estado, más no para las empresas. Un ejemplo de ello es el indicador de infraestructura vial, que si bien puede ser un indicador logístico, no está compuesto por factores sobre los que la empresa de transporte de carga puede influir. En este sentido, y dado que en las empresas de transporte de carga existen variables externas que afectan su desempeño como la demanda, la congestión vehicular, las restricciones para movilizar por ciertas rutas, etc., se hace necesario el uso de indicadores que permitan la toma de decisiones desde los diferentes niveles de la organización. Con el presente trabajo se busca proponer un marco metodológico que permita la elaboración de indicadores de desempeño que se basen en las actividades logísticas, que además de contener la definición de cada indicador incluya cómo calcularlos, enfocando la propuesta en las empresas de transporte de carga terrestre.

\section{ESTADO DEL ARTE}

La formulación de un indicador debe estar asociada al seguimiento de un objetivo, esto con la finalidad de que el indicador permita conocer el éxito o fracaso del objetivo a evaluar, su avance o retraso y señale las causales que permiten o impiden lograr el objetivo para identificar acciones que permitan corregirlas [6].

En [7] se analizan modelos para la evaluación del desempeño de la cadena de suministro, que si bien no es una metodología para desarrollar indicadores, resalta la necesidad de que estos modelos se apoyen en el uso de indicadores para la trazabilidad de la operación logística. En cuanto a las características de los indicadores, en [8] se presentan las condiciones a considerar al diseñar un indicador, el conjunto de estas características se resume en el criterio SMART por sus siglas en inglés (Specific, Measurable, Achievable, Realistic, Time- Bound), en la Figura 1 se explica la metodología SMART.

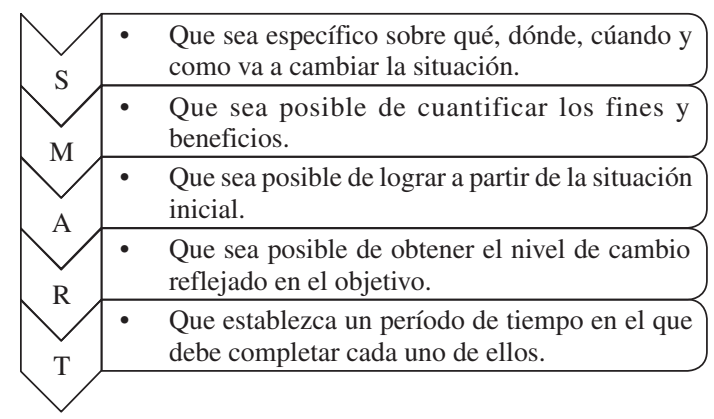

Figura 1. Características de un indicador SMART. 
Otros autores resaltan la necesidad de que los indicadores puedan ser representados numéricamente, es decir, que sean medibles y que la información requerida para medir esté disponible a un bajo costo [9]. En [6] se señala la importancia de que los indicadores formulados se basen en las características ya mencionadas; en [8]; sin embargo, se expresa la necesidad de considerar que el registro de los valores medidos por los indicadores puede no ser suficiente para realizar un análisis de las mejoras requeridas por la empresa, haciendo necesario plantear un plan de seguimiento a los indicadores donde se incluya un análisis situacional en cada medición realizada.

A la necesidad de realizar un análisis de las mejoras requeridas a partir del uso de indicadores mencionada en [6], ya en [10] se había propuesto un enfoque para analizar el cumplimiento de los indicadores de desempeño claves o KPI por sus siglas en inglés (Key Performance Indicators) en la cadena de suministro; en este enfoque, se propone un ciclo para la administración de indicadores que inicia con establecer los objetivos de la empresa para cada nivel de la organización; la segunda fase es establecer un modelo sobre el funcionamiento de la empresa que permita identificar los factores que afectan la operación, seguido de proponer un plan que incluya los indicadores que se desean implementar; posteriormente se propone un monitoreo para reunir la información, se reporta el estado de los indicadores y se proyecta el comportamiento de estos. Con base en lo anterior se analiza la información, para realizar un reporte de los resultados y se regresa a la primera etapa [10].

Teniendo claro qué es un indicador y sus características, se ha realizado una búsqueda de las medidas de desempeño para la cadena de suministro en general y para el transporte. En [5] se propone el tiempo de envío, el costo total, la satisfacción del cliente, y el costo ambiental como medidas de desempeño a considerar. De forma similar [11] presenta un marco metodológico para medir el desempeño de la cadena de suministro y en cuanto al transporte muestran como principales indicadores la satisfacción del cliente, tiempo de procesamiento y alistamiento del pedido y el costo total de distribución. En [12] usan un enfoque desde el Balanced Scorecard para formular medidas de desempeño de la cadena de suministro y en cuanto al transporte recomiendan indicadores como el tiempo de transporte, cumplir con las fechas de entrega, efectividad de las rutas y el costo de transporte.

En [13] se realiza un análisis de las métricas usadas en las cadenas de suministro sustentables; como indicadores resaltan las emisiones de $\mathrm{CO}_{2}$, la confiabilidad del servicio que está asociado a la satisfacción del cliente, el consumo de combustible y el costo total. Según [14] se debe considerar el efecto del uso de las Tecnologías de Información y Comunicación (TIC), en el desempeño del transporte, donde la principal hipótesis es la existencia de una relación positiva entre el uso de TIC y la disminución del tiempo de respuesta a los clientes, proponiendo implementar indicadores sobre el uso de tecnologías de la información en el transporte. En [15] se analizan indicadores referentes a la resiliencia de la empresa; el nivel de servicio y el costo de corregir los errores cometidos.

Tomando como un segundo enfoque de búsqueda los indicadores propuestos específicamente en el transporte, se encuentra en [16] un sistema de indicadores para el uso de entidades gubernamentales, aunque provee indicadores que pueden ser adaptados a las empresas de transporte como el factor de carga, consumo de combustible y los accidentes de tráfico. En [17] se identifican indicadores como el consumo de combustible, la accidentalidad, la calidad del servicio y el mantenimiento de los vehículos; mientras que en [18] se mide la eficiencia de las rutas en función del tiempo de viaje, la distancia entre destinos, el número de paradas realizadas, el consumo de combustible y la velocidad promedio. En cuanto a indicadores asociados al transporte de mercancía en cadenas de suministro específicas, en [19] se analizan los indicadores de competitividad en la cadena de suministro de la comida; incluyendo el número de empleados capacitados, el uso de combustible, el costo total de transporte, el número de ordenes despachadas sin errores y el cumplimiento con los tiempos.

De la búsqueda en literatura científica se encuentra que en los últimos años los indicadores ambientales y sociales han tomado fuerza en el análisis de la operación transporte, En [20] y [21] se estudia el comportamiento de indicadores como las emisiones de gases de efecto invernadero, el costo relacionado a la emisión de $\mathrm{CO}_{2}$, el número de accidentes de tránsito, niveles de contaminación auditiva, etc. Sin 
embargo, el uso de estos indicadores en las empresas de transporte de carga puede no ser viable, debido a que la obtención de la información para calcularlos puede resultar lenta y costosa.

En cuanto a investigaciones previas se encuentra que en 2014 el proyecto E-Transcol presenta un sistema de indicadores para el monitoreo del sistema logístico nacional; si bien presenta un enfoque para el seguimiento desde el estado hacia la operación transporte de carga, realiza una identificación de indicadores que pueden ser adoptados por las empresas privadas, haciendo énfasis en el seguimiento al procesamiento de órdenes de clientes, la planeación de inventarios y distribución. [22]

A partir de la búsqueda de documentos en organismos internacionales multilaterales que identifiquen indicadores logísticos, se encuentra por ejemplo el Banco Mundial que realiza un seguimiento al desempeño logístico de los países, aunque no se presenta indicadores enfocados en el sector privado, si proporciona a las empresas de transporte una visión de los componentes logísticos que deben considerar para aumentar su competitividad [23].

Los indicadores que se han propuesto en la literatura para hacer seguimiento a la cadena de suministro, sirven como base para identificar indicadores que para el análisis del desempeño en las empresas de transporte de carga en función de las actividades logísticas que se realizan al interior de las mismas, siendo estos indicadores logísticos que permiten la toma de decisiones en las diferentes áreas organizacionales.

\section{METODOLOGÍA DE LA INVESTIGACIÓN}

El trabajo de investigación que dio origen a este artículo "Estudio de los principales corredores logísticos en Colombia E-Transcol" permitió establecer una propuesta metodológica para la formulación de indicadores logísticos para las empresas de transporte de carga terrestre colombianas a partir de identificar: las áreas clave decisionales, las actividades logísticas y los recursos con que cuenta la organización y el conocimiento de las estrategias operativas y tácticas para generar los indicadores logísticos más adecuados para la organización. En la Figura 2 se muestra de forma esquemática dicha propuesta metodológica.

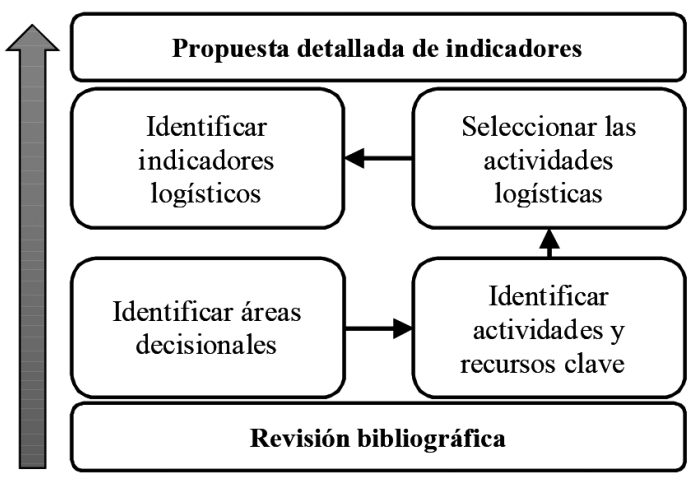

Figura 2. Propuesta metodológica para el desarrollo de indicadores logísticos.

La revisión bibliográfica es la etapa inicial de la presente metodología; allí se contempla la búsqueda en literatura científica, documentos gubernamentales nacionales e internacionales además de investigaciones previas que permitan el reconocimiento de indicadores logísticos propuestos para el sector. En esta etapa deben obtenerse indicadores logísticos al igual que una categorización de los mismos.

El segundo paso surge de la necesidad de implementar los indicadores propuestos en las empresas y es la identificación de las áreas decisionales; estas áreas decisionales se definen en función de la revisión bibliográfica o con paneles de expertos. Posteriormente, y en función de las áreas analizadas, se identifican las actividades y los recursos clave que influencian el desempeño de las empresas y responden a los procesos que se desarrollan en las áreas decisionales; sin embargo, la totalidad de estas actividades pueden no ser representativas para la logística en sí, por lo que el cuarto paso consiste en seleccionar el conjunto de tareas que apoyan los procesos logísticos al interior de la empresa o actividades logísticas, estas actividades pueden identificarse teniendo en cuenta que de su adecuada ejecución depende el éxito de la operación.

La identificación de los indicadores que permiten hacer seguimiento a la operación logística para las actividades seleccionadas es el siguiente paso, y se basa en las actividades logísticas seleccionadas, debe considerarse que los indicadores sean específicos, medibles, realistas y que sea posible establecer un período de tiempo para su seguimiento. 


\section{PROPUESTA PARA LA FORMULACIÓN DE INDICADORES}

A continuación, se describen los resultados obtenidos a partir de la ejecución metodológica presentada en la Figura 2; estos resultados finalizan con la propuesta de indicadores logísticos y permiten comprender la información que se debe obtener al seguir paso a paso esta metodología.

Inicialmente se presentan los indicadores identificados a partir de la revisión bibliográfica; la Tabla 1 presenta un resumen de los indicadores identificados que pueden ser implementados en las empresas de transporte de carga.

Tabla 1. Indicadores identificados en la revisión bibliográfica.

\begin{tabular}{|c|c|}
\hline Fuente & Indicadores \\
\hline$[5]$ & $\begin{array}{l}\text { - Tiempo de entrega. } \\
\text { - Costo total. } \\
\text { - Satisfacción del cliente. } \\
\text { - Costo ambiental. } \\
\end{array}$ \\
\hline$[11]$ & $\begin{array}{l}\text { - Satisfacción del cliente. } \\
\text { - Tiempo de procesamiento del pedido. } \\
\text { - Tiempo de alistamiento del pedido. } \\
\text { - Número de órdenes o facturas erróneas. } \\
\text { - Costo total de distribución. } \\
\text { - Costo asociado a los activos o retorno sobre } \\
\text { la inversión. }\end{array}$ \\
\hline$[12]$ & $\begin{array}{l}\text { - Tiempo de cumplimiento de una orden. } \\
\text { - Cumplimiento con las fechas de entrega. } \\
\text { - Efectividad de las rutas. } \\
\text { - Costo asociado al transporte. }\end{array}$ \\
\hline$[13]$ & $\begin{array}{l}\text { - Emisiones de } \mathrm{CO}_{2} \text {. } \\
\text { - Confiabilidad del servicio. } \\
\text { - Consumo de combustible. } \\
\text { - Costo total. }\end{array}$ \\
\hline [14] & $\begin{array}{l}\text { - Indicadores del uso de tecnologías de la } \\
\text { información }(T I) \text {. }\end{array}$ \\
\hline [15] & $\begin{array}{l}\text { - Nivel de servicio. } \\
\text { - Costo de corregir los errores cometidos. } \\
\text { - Número de despachos erróneos. }\end{array}$ \\
\hline [16] & $\begin{array}{l}\text { - Factor de carga. } \\
\text { - Consumo de combustible. } \\
\text { - Accidentes de tráfico. }\end{array}$ \\
\hline [17] & $\begin{array}{l}\text { - Consumo de combustible. } \\
\text { - Accidentalidad. } \\
\text { - Calidad del servicio. } \\
\text { - Mantenimiento de los vehículos. }\end{array}$ \\
\hline$[18]$ & $\begin{array}{l}\text { - Tiempo de viaje. } \\
\text { - Distancia entre destinos. } \\
\text { - Número de paradas realizadas. } \\
\text { - Consumo de combustible. } \\
\text { - Velocidad promedio. } \\
\text { - Características técnicas de los vehículos. } \\
\text { - Toneladas transportadas. }\end{array}$ \\
\hline
\end{tabular}

\begin{tabular}{|c|c|}
\hline Fuente & Indicadores \\
\hline [19] & $\begin{array}{l}\text { - Número de empleados capacitados. } \\
\text { - Indicador de logística inversa. } \\
\text { - Uso de combustible. } \\
\text { - Costo total de transporte. } \\
\text { - Número de órdenes despachadas sin errores. } \\
\text { - Validez del período de transporte. }\end{array}$ \\
\hline [22] & $\begin{array}{l}\text { - Velocidad promedio de tránsito. } \\
\text { - Gastos operacionales de los vehículos. } \\
\text { - Costo de transporte por tonelada-kilómetro. } \\
\text { - Tiempo total desde el punto de origen al de } \\
\text { destino. } \\
\text { - Tiempo de demora promedio. } \\
\text { - Disponibilidad de zonas logísticas. } \\
\text { - Inversión media en TIC. } \\
\text { - Vehículos que usan TIC. } \\
\text { - Número de sistemas de información de } \\
\text { servicios logísticos en operación. } \\
\text { - Porcentaje de transacciones de carga realizadas } \\
\text { vía web. }\end{array}$ \\
\hline [23] & $\begin{array}{l}\text { - Tiempo de ejecución de procedimientos } \\
\text { burocráticos. } \\
\text { - Uso de TIC. } \\
\text { - Cumplimiento con los tiempos de entrega. } \\
\text { - Robos. }\end{array}$ \\
\hline
\end{tabular}

La revisión bibliográfica permite además la detección de indicadores base con los que se evalúa el desempeño logístico del transporte, al igual que categorizarlos de forma precisa como se observa en la Tabla 2.

Tabla 2. Clasificación de indicadores logísticos encontrados.

\begin{tabular}{|c|c|}
\hline Categoría & Indicadores identificados \\
\hline Tiempos & $\begin{array}{l}\text { - Tiempo de procesamiento del pedido. } \\
\text { - Tiempo de respuesta a los clientes. } \\
\text { - Tiempos de consolidación y/o des- } \\
\text { consolidación. }\end{array}$ \\
\hline Costos & $\begin{array}{l}\text { - Costo de consolidación y/o de } \\
\text { desconsolidación. } \\
\text { - Costo total de transporte. } \\
\text { - Costo de corregir errores logísticos. } \\
\text { - Costo de combustible. }\end{array}$ \\
\hline Calidad & $\begin{array}{l}\text { - Nivel de satisfacción del cliente. } \\
\text { - Despachos erróneos. } \\
\text { - Número de empleados capacitados. }\end{array}$ \\
\hline Confiabilidad & $\begin{array}{l}\text { - Cumplimiento en el tiempo de entrega. } \\
\text { - Velocidad promedio. } \\
\text { - Trazabilidad o uso de las TI. } \\
\text { - Mantenimientos realizados. } \\
\text { - Accidentalidad. }\end{array}$ \\
\hline
\end{tabular}

\section{Identificación de las áreas decisionales}

Las áreas de decisión en las empresas han sido definidas en cuatro grupos; el primero de ellos es la 
capacidad y representa el volumen de producción o servicios prestados que puede lograrse con los recursos existentes. Tecnología de procesos comprende las decisiones sobre instalaciones, maquinaria, procesos, tecnologías de la información y posibles mejoras. La tercera área decisional es red de suministro y en esta se toman decisiones sobre la red entre proveedores, clientes, distribuidores, etc. Finalmente, el área de desarrollo y organización se encarga de gestionar las operaciones, tomando decisiones a largo y corto plazo para la mejora de procesos y la gestión de los recursos [24-26].

En cuanto a las empresas de transporte de carga, se encuentra en [27] una división de áreas decisionales que si bien responde a las áreas tradicionalmente establecidas, proveen una clasificación agrupada de las mismas; la primera área decisional propuesta es producto o servicio donde se analizan los cambios que se requieren para minimizar costos y mejorar el servicio prestado; la segunda área es la de distribución donde se deben tomar acciones para reducir costos por medio de la asignación de rutas, camiones y disminuyendo el número de transacciones; finalmente, definen el área de clientes que define las estrategias para mejorar el servicio prestado.

En la gestión de la cadena de suministro se requiere planear estratégicamente a través de la gerencia de las empresas [28], en función de estos requerimientos y las áreas decisionales ya propuestas en la literatura científica; se definen para la presente propuesta metodológica cuatro áreas decisionales a considerar:

- Gerencia: Esta es la encargada de generar y velar por el cumplimiento de las políticas organizacionales, debe ser un vínculo entre las fuentes de información externas a la empresa y las demás áreas. Dirige el funcionamiento de la empresa y toma decisiones en el corto y largo plazo.

- Soporte administrativo: Esta área apoya la toma de decisiones al área decisional definida como desarrollo y organización, donde se consideran actividades como el servicio al cliente, ventas, compras, contabilidad y recursos humanos.

- Operaciones -Logística-: Análoga a las áreas decisionales de capacidad y red de suministro, es desde esta área donde dónde se toman las decisiones sobre el despacho, rutas y vehículos a usar, debe estar enterado de las restricciones en la operación y coordinar el mantenimiento preventivo y correctivo de los equipos.

- Tecnología de procesos: Esta área es la encargada de tomar decisiones sobre la implementación y administración de tecnologías de información, instalaciones, maquinaria, procesos y servicios prestados.

La identificación de áreas decisionales, puede también realizarse usando el método Delphi [29], este método permite establecer respuestas a una pregunta planteada por medio de la experticia de personas que han trabajado en torno al problema que se plantee [30]; en este caso serían personas que pertenecen a empresas de transporte de carga y que conocen trasversalmente el funcionamiento de las mismas.

En la Figura 3, las Hojas de Nominación consideran las habilidades que deben tener los expertos ya sean académicos, empresarios e incluso expertos que pertenezcan a instituciones gubernamentales oficiales que ofrecen una visión global de lo que se espera de las empresas de transporte; en función de estas necesidades se completan las hojas de nominación; posteriormente se deben puntuar los expertos para así contactar e invitar a aquellos con mayor puntaje [29-31]. La Figura 3 ilustra los pasos para la selección de las áreas decisionales usando el método Delphi.

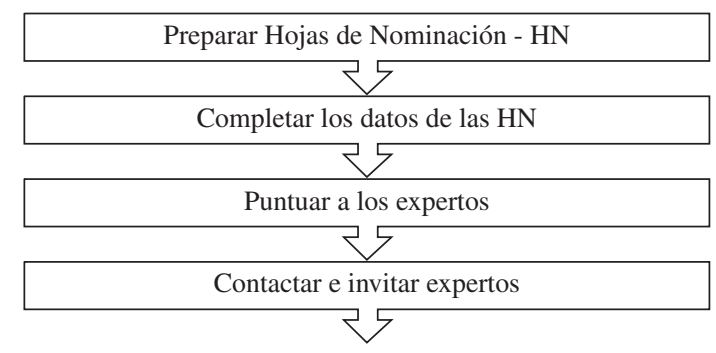

Figura 3. Pasos método Delphi para identificar áreas decisionales.

\section{Identificación de actividades y recursos clave}

En la Tabla 3 se presentan las actividades que se consideran clave y deben desarrollarse al interior de cada área decisional. 
Tabla 3. Identificación de actividades clave.

\begin{tabular}{|c|c|}
\hline Área decisional & Actividad clave \\
\hline \multirow{7}{*}{ Gerencia } & $\begin{array}{l}\text { Hacer seguimiento a los indicadores } \\
\text { de desempeño. }\end{array}$ \\
\hline & $\begin{array}{l}\text { Proponer la generación de indicadores } \\
\text { de gestión. }\end{array}$ \\
\hline & $\begin{array}{l}\text { Realizar alianzas estratégicas con } \\
\text { proveedores y clientes. }\end{array}$ \\
\hline & $\begin{array}{l}\text { Ejecutar acciones correctivas a } \\
\text { problemas identificados en la } \\
\text { organización. }\end{array}$ \\
\hline & $\begin{array}{l}\text { Hacer seguimiento al tiempo de ciclo } \\
\text { de la operación. }\end{array}$ \\
\hline & $\begin{array}{l}\text { Evaluar el compromiso de los } \\
\text { empleados. }\end{array}$ \\
\hline & $\begin{array}{l}\text { Evaluar el estado operativo de los } \\
\text { recursos físicos de la empresa. }\end{array}$ \\
\hline \multirow{5}{*}{$\begin{array}{l}\text { Soporte } \\
\text { administrativo }\end{array}$} & $\begin{array}{l}\text { Documentar y archivar información } \\
\text { relativa al servicio: Ventas, quejas, } \\
\text { compras, etc. }\end{array}$ \\
\hline & $\begin{array}{l}\text { Realizar registro y control de las } \\
\text { finanzas. }\end{array}$ \\
\hline & Capacitar RRHH. \\
\hline & Prestar servicio al cliente. \\
\hline & Mercadeo. \\
\hline \multirow{7}{*}{$\begin{array}{l}\text { Operaciones } \\
\text {-Logística- }\end{array}$} & Coordinar mantenimientos. \\
\hline & Asignaciín de rutas y vehículos. \\
\hline & Despachar. \\
\hline & Controlar indicadores logísticos. \\
\hline & Prestar servicio al cliente. \\
\hline & $\begin{array}{l}\text { Realizar informes sobre el desempeño } \\
\text { del área. }\end{array}$ \\
\hline & $\begin{array}{l}\text { Comunicar requerimientos operacio- } \\
\text { nales a soporte administrativo. }\end{array}$ \\
\hline \multirow{5}{*}{$\begin{array}{l}\text { Tecnología de } \\
\text { procesos }\end{array}$} & $\begin{array}{l}\text { Realizar informes sobre el estado de } \\
\text { la operación. }\end{array}$ \\
\hline & Identificar problemas. \\
\hline & Realizar trazabilidad a los datos. \\
\hline & $\begin{array}{l}\text { Procesar la información enviada } \\
\text { desde las demás áreas. }\end{array}$ \\
\hline & $\begin{array}{l}\text { Identificar las necesidades del } \\
\text { mercado y las capacidades de la } \\
\text { empresa para satisfacerlas. }\end{array}$ \\
\hline
\end{tabular}

En la gerencia se identifican siete actividades enfocadas en el desarrollo de estrategias que permitan hacer trazabilidad a los indicadores y en el desempeño general de la empresa; en el área de soporte administrativo se identifican actividades clave que se relacionan con la ejecución de las estrategias planteadas desde la gerencia, incluyendo el servicio al cliente como actividad crítica en cualquier empresa [2]; [32]; [33]. En el área de relaciones se identifican actividades operativas que se relacionan directamente con la prestación del servicio de transporte. Por su parte el área de tecnología de procesos presenta actividades relacionadas a la gestión de la información desde la trazabilidad a los datos [34]; la toma de decisiones para la solución oportuna de problemas [35]; y actividades que aportan a las estrategias de la empresa desde la definición de las necesidades del mercado y la capacidad del negocio [36]; [37-38].

\section{Selección de actividades logísticas}

Los procesos logísticos al interior de las empresas de transporte de carga se ven afectados por la totalidad de actividades que se realizan en las diferentes áreas, presentándose una dependencia multidireccional entre ellas [39]. La Tabla 4 presenta las actividades logísticas identificadas en las diferentes áreas decisionales.

Tabla 4. Selección de actividades logísticas en las áreas decisionales.

\begin{tabular}{|l|l|}
\hline Área decisional & \multicolumn{1}{c|}{ Actividades logísticas } \\
\hline \multirow{5}{*}{ Gerencia } & $\begin{array}{l}\text { Hacer seguimiento a los indicadores } \\
\text { de desempeño. }\end{array}$ \\
\cline { 2 - 2 } & $\begin{array}{l}\text { Hacer seguimiento al tiempo de ciclo } \\
\text { de la operación. }\end{array}$ \\
\cline { 2 - 2 } & $\begin{array}{l}\text { Evaluar el compromiso de los } \\
\text { empleados. }\end{array}$ \\
\cline { 2 - 2 } & $\begin{array}{l}\text { Evaluar el estado operativo de los } \\
\text { recursos físicos de la empresa. }\end{array}$ \\
\hline \multirow{2}{*}{$\begin{array}{l}\text { Soporte } \\
\text { administrativo }\end{array}$} & Capacitar RRHH. \\
\cline { 2 - 2 } & Prestar servicio al cliente. \\
\hline \multirow{4}{*}{$\begin{array}{l}\text { Operaciones }- \text { Logística- } \\
\text { Coordinar mantenimientos. }\end{array}$} & Asignar de rutas y vehículos. \\
\cline { 2 - 2 } & Despachar. \\
\cline { 2 - 2 } & Controlar indicadores logísticos. \\
\cline { 2 - 2 } & Prestar servicio al cliente. \\
\hline \multirow{2}{*}{$\begin{array}{l}\text { Tecnología de } \\
\text { procesos }\end{array}$} & Identificar problemas. \\
\cline { 2 - 2 } & Procesamiento de la información. \\
\hline
\end{tabular}

En la gerencia se identifican actividades necesarias para controlar el desempeño de la operación; evaluar el compromiso de empleados permite identificar fallas organizacionales que disminuyen la productividad; también se hace necesario realizar seguimiento de la operación, a los indicadores logísticos y evaluar el estado operativo de los recursos físicos. En soporte administrativo se propone la capacitación de los recursos humanos, 
ya que contar con personal especializado permite mejorar el desempeño de la empresa. Prestar servicio al cliente es una actividad que se asocia tanto al área de soporte administrativo como al área de operaciones. Como actividades del área de operaciones se identifican la asignación de rutas y vehículos, coordinar mantenimientos, el despacho de pedidos y el control de los indicadores logísticos [38]. El área de tecnología de procesos se asocia con procesar la información e identificar problemas.

\section{Selección de indicadores logísticos}

En función de los resultados obtenidos de la búsqueda bibliográfica, se identifican los indicadores logísticos a considerar en las áreas decisionales, obteniendo:

- Gerencia: Seguimiento a indicadores, tiempo promedio total para finalizar una orden, satisfacción de los empleados, rotación del personal, edad del parque automotor.
- Soporte administrativo: Personal capacitado, clientes inconformes, quejas, fidelidad de los clientes, tiempo promedio para el procesamiento de una orden de compra.

- Área Operaciones-Logística-: Mantenimientos realizados, costo de actividades logísticas, velocidad promedio, factor de carga, tiempo de procesamiento de pedido, tiempo de consolidar y/o desconsolidar, tiempo de entrega cumplido.

- Tecnología de procesos: Accidentalidad, implementación de TIC, indicadores para las causas de insatisfacción de los clientes e indicadores para el análisis de los costos.

\section{Propuesta detallada de indicadores}

Como resultado final del análisis realizado se describe detalladamente cada indicador. En la Tabla 5 se identifica el nivel organizacional al que pertenece

Tabla 5. Propuesta de indicadores por área decisional.

\begin{tabular}{|c|c|c|c|}
\hline Nivel & Área decisional & Actividad clave & Indicador propuesto \\
\hline \multirow{4}{*}{ Estratégico } & \multirow{4}{*}{ 1. Gerencia } & $\begin{array}{l}\text { Hacer seguimiento a los } \\
\text { indicadores de desempeño. }\end{array}$ & 1.1. Seguimiento a indicadores. \\
\hline & & $\begin{array}{l}\text { Hacer seguimiento al tiempo } \\
\text { de ciclo de la operación. }\end{array}$ & $\begin{array}{l}\text { 1.2. Tiempo promedio total para finalizar una orden } \\
\text { (Tiempo promedio de respuesta). }\end{array}$ \\
\hline & & $\begin{array}{l}\text { Evaluar el compromiso de } \\
\text { los empleados. } \\
\end{array}$ & $\begin{array}{l}\text { 1.3. Satisfacción de los empleados. } \\
\text { 1.4. Rotación del personal. }\end{array}$ \\
\hline & & $\begin{array}{l}\text { Evaluar el estado operativo } \\
\text { de los recursos físicos de la } \\
\text { empresa. }\end{array}$ & 1.5. Edad promedio de los vehículos. \\
\hline \multirow[b]{2}{*}{ Táctico } & \multirow[b]{2}{*}{$\begin{array}{l}\text { 2. Soporte } \\
\text { administrativo }\end{array}$} & Capacitación RRHH. & 2.1. Porcentaje de personal capacitado. \\
\hline & & Prestar servicio al cliente. & $\begin{array}{l}\text { 2.2. Porcentaje de clientes inconformes. } \\
\text { 2.3. Quejas presentadas. } \\
\text { 2.4. Porcentaje de clientes que contratan nuevamente } \\
\text { el servicio. }\end{array}$ \\
\hline \multirow{4}{*}{ Operativo } & \multirow{4}{*}{$\begin{array}{l}\text { 3. Operaciones } \\
\text {-Logística- }\end{array}$} & Coordinar mantenimientos. & 3.1. Cumplimiento a los mantenimientos programados. \\
\hline & & $\begin{array}{l}\text { Asignación de rutas y } \\
\text { vehículos. }\end{array}$ & $\begin{array}{l}\text { 3.2. Costo promedio de transporte por tonelada. } \\
\text { 3.3. Uso de los vehículos. } \\
\text { 3.4. Factor de carga promedio. } \\
\text { 3.5. Velocidad promedio de los vehículos. }\end{array}$ \\
\hline & & Despachar & $\begin{array}{l}\text { 3.6. Tiempo de procesamiento logístico. } \\
\text { 3.7. Tiempo consolidación y /o desconsolidación. }\end{array}$ \\
\hline & & Servicio al cliente. & 3.8. Retrasos en la entrega. \\
\hline \multirow[b]{2}{*}{ Táctico } & \multirow[b]{2}{*}{$\begin{array}{l}\text { 4. Tecnología de } \\
\text { procesos }\end{array}$} & $\begin{array}{l}\text { Procesamiento de la } \\
\text { información. }\end{array}$ & 4.1. Uso de TIC. \\
\hline & & $\begin{array}{l}\text { Identificar posibles problemas } \\
\text { y proponer soluciones. }\end{array}$ & $\begin{array}{l}\text { 4.2. Accidentalidad. } \\
\text { 4.3. Causas de insatisfacción de los clientes. } \\
\text { 4.4. Costos reales asumidos. }\end{array}$ \\
\hline
\end{tabular}


cada grupo de indicadores, el área decisional y la actividad clave a la que está asociado.

A continuación se describen los indicadores mencionados en la Tabla 4 para cada área decisional conservando la numeración propuesta.

\section{GERENCIA}

\subsection{Seguimiento a Indicadores - SI}

Los gerentes deben establecer un objetivo en el tiempo de revisión a los indicadores, lo ideal obtener valores cercanos al 100\%, la ecuación (1) presenta la fórmula para calcular este indicador, donde NSR son el número de seguimientos realizados y NSP el número de seguimientos programados.

$$
S I=\frac{N S R}{N S P} \times 100
$$

\subsection{Tiempo Promedio de Respuesta - TRP}

Desde la gerencia se debe establecer el tiempo promedio total máximo para finalizar una orden. El área de tecnología de procesos debe establecer los tiempos que la competencia ha establecido para así brindar un marco de referencia a la gerencia. El cálculo del tiempo promedio de respuesta lo presenta la ecuación (2), donde $n$ es el número de órdenes de compra procesadas en el período de estudio y TROCi es el tiempo de respuesta a la orden de compra $i$.

$$
T P R=\frac{\sum_{i=1}^{i=n} T_{R O C_{i}}}{n}
$$

\subsection{Satisfacción de los Empleados - SE}

Para medir este indicador se propone realizar una encuesta de carácter anónimo, en esta encuesta se realizarían preguntas respondidas con una escala de Likert (1 a 10) y diferenciadas por factores del clima organizacional que afectan la motivación, a cada uno de estos factores se le desarrollarían preguntas sobre la satisfacción del personal, las respuestas por cada factor se ponderan obteniendo una calificación media para cada empleado encuestado; de allí se procede a cuantificar el porcentaje de empleados satisfechos en el factor $i$. La ecuación (3) presenta el cálculo de este indicador, donde $N E C S$ es el número de empleados que calificaron satisfactoriamente (sobre 7) y $N E E$ el número de empleados encuestados. Se espera maximizar el valor arrojado por el indicador.

$$
S E_{i}=\frac{N E C S}{N E E} \times 100
$$

\subsection{Rotación del Personal - $\boldsymbol{R} P$}

Este indicador permite conocer la estabilidad de la nómina, altos valores de rotación pueden significar pérdida de conocimiento y disminución en la productividad. La ecuación 4 expresa el cálculo de este indicador, donde $E S$ número de empleados que salieron de la empresa en el período de estudio, mientras que $E E$ es el número de empleados entrantes en el mismo período. $E I$ es el número de empleados al inicio del período y $E F$ es el número de empleados registrados al final.

$$
R P=\frac{\frac{E S+E E}{2}}{\frac{E I+E F}{2}} \times 100
$$

\subsection{Edad Promedio de los Vehículos - EPV}

Es el gobierno quien establece las edades permitidas de los vehículos usados, por lo que deben establecerse valores que estén acordes con la ley. La ecuación (5) presenta el cálculo de este indicador, donde $E V_{i}$ es la edad del vehículo $i$, y $n$ es el número total de vehículos.

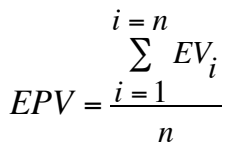

\section{SOPORTE ADMINISTRATIVO}

\subsection{Porcentaje de Personal Capacitado - PPC}

$\mathrm{El}$ indicador de recurso humano capacitado permite mejorar el nivel de servicio prestado al cliente, las condiciones laborales de los conductores se disminuyen los riesgos por accidentalidad, etc. Se espera maximizar el porcentaje de personal capacitado, el cálculo se presenta en la ecuación (6), donde $P C$ es el personal capacitado y $P T$ el personal total.

$$
P P C=\frac{P C}{P T}
$$




\subsection{Porcentaje de Clientes Inconformes - PCI}

Para medir este indicador es necesario presentar una encuesta al finalizar el servicio de transporte. La escala de medición puede basarse en una escala de Likert de 1 a 5 ; siendo 1 muy inconforme; 2 inconforme, 3 indiferente 4 satisfecho y 5 muy satisfecho. La ecuación (7) presenta el cálculo de este indicador.

$$
P C I=\frac{N C I}{C E}
$$

Donde NCI es el número de clientes inconformes y $C E$ el número de clientes encuestados.

\subsection{Quejas Presentadas - $Q P$}

A diferencia del de porcentaje de clientes inconformes, se obtiene a partir de las quejas que los clientes presentan sin necesidad de registrarlo en una encuesta, se busca minimizar el valor obtenido al calcular el indicador, el cálculo de este indicador se presenta en la ecuación (8), donde $N Q P$ es el número de quejas presentadas y $N O R$ el número de operaciones realizadas.

$$
Q P=\frac{N Q P}{N O R}
$$

\subsection{Porcentaje de Clientes que Contratan Nuevamente el Servicio - PCR}

Este indicador mide la lealtad de los clientes hacia la empresa. Se espera aumentar el valor obtenido, la ecuación (9) presenta el cálculo del indicador, donde $N C R$ es el número de clientes que recontrataron y $N T C R$ el número total de clientes registrados.

$$
P C R=\frac{N C R}{N T C R}
$$

\section{OPERACIONES -LOGÍSTICA-}

\subsection{Porcentaje de Cumplimiento a Mantenimientos Programados - PCMP}

Con este indicador se pretende medir el nivel de cumplimiento de los mantenimientos programados para un período de análisis; lo ideal es cumplir con los mantenimientos programados, por lo que se espera maximizar el valor obtenido. La ecuación (10) presenta el cálculo de este indicador donde
$M R$ son los mantenimientos realizados y $M P$ los mantenimientos programados.

$$
P C M P=\frac{M R}{M P}
$$

\subsection{Costo Promedio de Transporte por Tonelada - CPTT}

El valor estimado de este indicador se debe definir con apoyo del área de tecnología de procesos, quienes deben identificar los valores promedios del sector; su cálculo se presenta en la ecuación (11):

$$
C P T T=\frac{\sum_{i=1}^{i=n} C T_{i}}{\sum_{i=1}^{i=n} T_{i}}
$$

Donde $C T$ es el costo total en la operación $i$, y $T_{i}$ representa las toneladas totales movilizadas en la operación $i$; siendo $n$ el número de operaciones realizadas en el período de medición.

\subsection{Uso de los Vehículos - $U V$}

Este indicador está asociado a las horas efectivas de uso de un vehículo para transportar mercancía; el número de horas es un factor determinado por la empresa de transporte; se espera que sea 10 horas día, ya que es el tiempo que un conductor puede laborar sin poner en riesgo su salud y la mercancía. Lo ideal es maximizar el valor que arroja el indicador, de lo contrario se analiza la posibilidad de tener una sobre oferta vehicular. La ecuación (12) presenta el cálculo de este indicador, donde $H V O$ son las horas que el vehículo está ocupado y $H U D V$ el número de horas útiles disponibles del vehículo.

$$
U V=\frac{H V O}{H U D V} \times 100
$$

\subsection{Factor de Carga Promedio - FCP}

Permite conocer el verdadero uso de la capacitad instalada, aunque el indicador uso de vehículo que sea alto, puede que los vehículos se estén movilizando con mínima carga. Se debe buscar maximizar el valor arrojado por este indicador, la ecuación (13) presenta el cálculo de este indicador, donde $T M$ son las toneladas movilizadas, $C T V$ es la capacidad total de los vehículos en toneladas y $N V$ el número de viajes. 


$$
F C P=\frac{\frac{T M}{C T V}}{N V}
$$

\subsection{Velocidad Promedio de los Vehículos - VPV}

Este indicador busca medir la velocidad a que los vehículos transitan, el valor de referencia se establece a partir de los estándares del sector y que deben ser identificados por el área de tecnología de procesos. A partir del seguimiento a la velocidad promedio, puede identificarse si las rutas establecidas desde el área operativa están presentando problemas. El cálculo de este indicador se presenta en la ecuación (14).

$$
V P V=\frac{\sum_{i=1}^{i=n} \bar{V}_{i}}{n}
$$

\subsection{Cumplimiento del Tiempo de Procesamiento Logístico - CTPL}

Hace seguimiento a la respuesta que desde el área operacional-logística-se está da a los requerimientos presentados desde el soporte administrativo; el tiempo de referencia, $T R$; para completar un pedido se establece desde la gerencia buscando maximizar el valor arrojado por el indicador. La ecuación (15) presenta el cálculo de este indicador, donde $T P P_{i}$ es el tiempo para procesar el pedido $i, n$ es el número total de pedidos procesados en el período de medición y $T R$ el tiempo de referencia.

$$
C T P L=\frac{\sum_{i=1}^{i=n} T P P_{i}}{n}
$$

\subsection{Cumplimiento al Tiempo de Consolidación o Desconsolidación - CTC/ CTD}

Su cálculo se muestra en las ecuaciones (16) y (17), Donde CTC es el cumplimiento al tiempo de consolidación, $C T D$ es el cumplimiento al tiempo de desconsolidación, $T C_{i}$ y $T D_{i}$ son el tiempo de consolidación y desconsolidación para el pedido $i$, $n$ es el número de pedidos procesados en el período de medición, y el TRC y TRD son los tiempos de referencia para consolidar y desconsolidar definido por la gerencia con apoyo del área operativa. Se espera maximizar el valor calculado.

$$
C T C=\frac{\sum_{i=1}^{i=n} T C_{i}}{\operatorname{TRC}}
$$

$$
C T P L=\frac{\sum_{i=1}^{i=n} T D_{i}}{\operatorname{TRD}}
$$

\subsection{Retrasos en la Entrega - $R E$}

Es la relación entre tiempo real de entrega y la diferencia con el tiempo estimado; se espera que este indicador se minimice en función del nivel de servicio que la empresa desea establecer. La ecuación (18) muestra el cálculo de este indicador, donde TEE es el tiempo estimado de entrega y TRE el tiempo real de entrega.

$$
R E=\frac{T E E}{T R E}
$$

\section{TECNOLOGÍA DE PROCESOS}

\subsection{Uso de $T I C$ - UTIC}

Refleja el uso de TIC para la trazabilidad de la operación; se esperaría maximizar el valor de este indicador con el tiempo, la ecuación (19) presenta el cálculo de este indicador, donde NVTIC es el número de vehículos que usan TIC y NTV es el número total de vehículos.

$$
U T I C=\frac{N V T I C}{N T V}
$$

\subsection{Accidentalidad - $A C C$}

Mide la cantidad de incidentes que presentan los vehículos durante un período determinado definido por la empresa; la ecuación (20) presenta el cálculo de este indicador.

$$
A C C=\frac{N I}{N V R}
$$

\subsection{Causas de Insatisfacción de los Clientes}

Inicialmente se deben definir las causas de quejas, a cada una de las causas se le aplicaría el indicador 
insatisfacción del cliente asociado a la causa $i I C_{i}$ como lo muestra la ecuación (21); donde $Q_{i}$ es el número de quejas reportadas asociadas a la causa $i$ en un período y $Q T$ son las quejas totales reportadas en el mismo período.

$$
I C_{i}=\frac{Q_{i}}{Q T} \times 100
$$

\subsection{Costos Reales Asumidos - CRA}

Refleja la diferencia entre el costo estimado de realizar un viaje, y el costo real asumido al finalizar la actividad; este indicador acumula el total de costos estimados para cada viaje y los costos reales asumidos en un período. Se espera que el valor de este indicador se maximice, su cálculo se muestra en la ecuación (22), donde $C E O$ es el costo estimado de la operación y $C R A O$ es el costo real asumido en la operación.

$$
C R A=\frac{C E O}{C R A O}
$$

\section{CONCLUSIONES}

De la ejecución sistemática de la propuesta para el desarrollo de indicadores para las empresas de transporte terrestre de carga, es posible obtener un conjunto de métricas que consideren las necesidades identificadas en literatura científica y por expertos en transporte, sin obviar las particularidades de las empresas, asegurando la funcionalidad y el éxito de estos indicadores para medir el desempeño de las empresas.

La propuesta metodológica presentada para el desarrollo de indicadores útiles en las empresas de transporte de carga terrestre es novedosa y es trasversal, ya que considera la relación entre los diferentes niveles organizacionales, áreas decisionales y actividades clave para la construcción de medidas de desempeño que respondan a las condiciones reales de las empresas, permitiendo identificar las actividades que no se están ejecutando eficientemente, y así generar estrategias para mejorar los valores obtenidos de los indicadores.

La definición de los indicadores resultantes de seguir la metodología propuesta, debe permitir identificar las áreas decisionales que desde la empresa plantearan los valores esperados para cada indicador; la implantación de los indicadores propuestos debe además considerar el uso de tecnologías de información que faciliten el cálculo automático de las métricas e informen oportunamente las actividades que no cumplen con los valores estándar definidos por la empresa.

\section{AGRADECIMIENTOS}

Los autores agradecen al Departamento Administrativo de Ciencia, Tecnología e Innovación (COLCIENCIAS) y al Ministerio de Transporte por apoyar la investigación que ha dado origen a este artículo por medio de la Convocatoria 603 de 2012. El proyecto del cual ha surgido este artículo como resultado de investigación, lleva por título "Estudio del sistema logístico de carga en los principales corredores de Colombia - E-Transcol", identificado con el código 111860337707 de Colciencias. Adicionalmente, los autores agradecen a la Universidad Nacional de Colombia y el Departamento Administrativo para la Ciencia, Tecnología e Innovación (COLCIENCIAS), quienes mediante la convocatoria 617 de 2013 financiaron el desarrollo del proyecto de investigación "Herramientas de ingeniería para optimización de los recursos en el sistema de transporte de carga colombiano".

\section{REFERENCIAS}

[1] Departamento Nacional de Planeación. Guía Metodológica para la Formulación de Indicadores, pp. 4-5. 2010. Fecha de consulta: Julio de 2015. URL: http://www. bogota.unal.edu.co/planeacion/download/ documentos-enlaces/DNP\%20Guia\%20 Metodologica $\% 20$ Formulacion $\% 20-\% 20$ 2010.pdf

[2] H. Forslund. "The impact of performance management on customers' expected logistics performance". Int. J. Oper. Prod. Manag. Vol. $27 \mathrm{~N}^{\circ} 8$, pp. 901-918. 2007. DOI: 10.1108/01443570710763822

[3] P. Ahi and C. Searcy. "An analysis of metrics used to measure performance in green and sustainable supply chains". J. Clean. Prod. Vol. 86, pp. 360-377. 2015. DOI: 10.1016/j. jclepro.2014.08.005.

[4] A. Mascarenhas, L. M. Nunes and T.B. Ramos. "Selection of sustainability indicators 
for planning: combining stakeholders participation and data reduction techniques". J. Clean. Prod. Vol. 92, pp. 295-307. 2015. DOI: $10.1016 /$ j.jclepro.2015.01.005

[5] K.R.W. Azfar, N. Khan and H.F. Gabriel. "Performance Measurement: A Conceptual Framework for Supply Chain Practices". Procedia - Soc. Behav. Sci. Vol. 150, pp. 803812. 2014. DOI: 10.1016/j.sbspro.2014.09.089

[6] J. Woxenius. "Directness as a key performance indicator for freight transport chains". Res. Transp. Econ. Vol. 36 N $^{\circ}$ 1, pp. 63-72. 2012. DOI: 10.1016/j.retrec.2012.03.007

[7] D. Estampe, S. Lamouri, J.-L. Paris and S. Brahim-Djelloul. "A framework for analysing supply chain performance evaluation models". Int. J. Prod. Econ. Vol. $142 \mathrm{~N}^{\circ}$ 2, pp. 247-258. 2013. DOI: $10.1016 /$ j.ijpe.2010.11.024

[8] P. Drucker. "The Practice of Management". Harpercollins Publishers. 3rd ed. New York, Estados Unidos, pp. 404. 2006. ISBN: 9780060878979

[9] H. Castillo and D. E. Pitfield. "ELASTIC-A methodological framework for identifying and selecting sustainable transport indicators". Transp. Res. Part Transp. Environ. Vol. 15 $\mathrm{N}^{\mathrm{o}}$ 4, pp. 179-188. 2010. DOI: 10.1016/j. $\operatorname{trd} .2009 .09 .002$

[10] J. Cai, X. Liu, Z. Xiao and J. Liu. "Improving supply chain performance management: A systematic approach to analyzing iterative KPI accomplishment". Decis. Support Syst. Vol. $46 \mathrm{~N}^{\circ} 2$, pp. 512-521. 2009. DOI: 10.1016/j.dss.2008.09.004

[11] A. Gunasekaran, C. Patel and R.E. McGaughey. "A framework for supply chain performance measurement". Int. J. Prod. Econ. Vol. 87 $\mathrm{N}^{\circ} 3$, pp. 333-347. 2004. DOI: 10.1016/j. ijpe.2003.08.003

[12] R. Bhagwat and M.K. Sharma. "Performance measurement of supply chain management: A balanced scorecard approach". Comput. Ind. Eng. Vol. $53 \mathrm{~N}^{\circ}$ 1, pp. 43-62. 2007. DOI: $10.1016 /$ j.cie.2007.04.001

[13] E. Hassini, C. Surti and C. Searcy. "A literature review and a case study of sustainable supply chains with a focus on metrics". Int. J. Prod. Econ. Vol. $140 \mathrm{~N}^{\circ} 1$, pp. 69-82. 2012. DOI: 10.1016/j.ijpe.2012.01.042

[14] A.Z. Acar and M.B. Uzunlar. "The Effects of Process Development and Information
Technology on Time-based Supply Chain Performance". Procedia - Soc. Behav. Sci. Vol. 150, pp. 744-753. 2014. DOI: 10.1016/j. sbspro.2014.09.044

[15] S.R. Cardoso, A.P.F. D. Barbosa-Póvoa and S. Relvas. "Design and planning of supply chains with integration of reverse logistics activities under demand uncertainty". Eur. J. Oper. Res. Vol. 226 No 3, pp. 436-451. 2013. DOI: $10.1016 /$ j.ejor.2012.11.035

[16] T.-A. Shiau and J.-S. Liu. "Developing an indicator system for local governments to evaluate transport sustainability strategies". Ecol. Indic. Vol. 34, pp. 361-371. 2013. DOI: 10.1016/j.ecolind.2013.06.001

[17] T.W. Smith, C.J. Axon and R.C. Darton. "A methodology for measuring the sustainability of car transport systems". Transp. Policy. Vol. 30, pp. 308-317. 2013. DOI: 10.1016/j. tranpol.2013.09.019

[18] J. Castanedo, M.Á. Pesquera, P. CasaresHontañón, P. Coto-Millan and V. Borissov. "Efficient Route of Freight Transport by Road, Evaluated with Innotransmer". Procedia - Soc. Behav. Sci. Vol. 160, pp. 634-643. 2014. DOI: $10.1016 /$ j.sbspro.2014.12.177.

[19] A. Turi, G. Goncalves, and M. Mocan. "Challenges and Competitiveness Indicators for the Sustainable Development of the Supply Chain in Food Industry". Procedia - Soc. Behav. Sci. Vol. 124, pp. 133-141. 2014. DOI: 10.1016/j.sbspro.2014.02.469

[20] I. Gallego-Álvarez and J. L. VicenteVillardón. "Analysis of environmental indicators in international companies by applying the logistic biplot". Ecol. Indic., Vol. 23, pp. 250-261. 2012. DOI: 10.1016/j. ecolind.2012.03.024

[21] K. Kijewska and B.G. Johansen. "Comparative Analysis of Activities for More Environmental Friendly Urban Freight Transport Systems in Norway and Poland". Procedia - Soc. Behav. Sci. Vol. 151, pp. 142-157. 2014. DOI: 10.1016/j.sbspro.2014.10.015

[22] Documento de Investigación. "Definición de un sistema de indicadores para el monitoreo del Sistema Logístico Nacional". $\mathrm{N}^{\circ}$ 7. Universidad Nacional de Colombia. Unpublished. 2014.

[23] World Bank. Connecting to Compete, pp. 1-5. 2014. Fecha de consulta: Julio 2015. URL: 
http://www.worldbank.org/content/dam/ Worldbank/document/Trade/LPI2014.pdf

[24] N. Slack and M. Lewis. "Operations Strategy". Prentice Hall. 3rd ed, pp. 459. Londres, Reino Unido. 2011. ISBN: 978-0-273-74044-5

[25] T.H. Davenport. "Process innovation". Harvard Business Press. 5th ed. Boston, Estados Unidos, pp. 352. 1992. ISBN: 0-87584-366-2

[26] D. Collis and C. Montgomery. "Corporate Strategy: Resources and The Scope of The Firm", McGraw-Hill. 1st ed, pp. 784. Boston, Estados Unidos. 1997. ISBN: 978-0-256-17894-4

[27] M. Hesse. "Shipping news: the implications of electronic commerce for logistics and freight transport". Resour. Conserv. Recycl. Vol. $36 \mathrm{~N}^{\mathrm{o}} 3$, pp. 211-240. 2002. DOI: 10.1016/S0921-3449(02)00083-6

[28] M. Hesse and J.-P. Rodrigue. "The transport geography of Logistics and Freight Distribution". J. Transp. Geogr. Vol. 12 $\mathrm{N}^{\mathrm{o}}$ 3, pp. 171-184. 2004. DOI: 10.1016/j. jtrangeo.2003.12.004

[29] C. Okoli and S. D. Pawlowski. "The Delphi method as a research tool: an example, design considerations and applications". Inf. Manage. Vol. $42 \mathrm{~N}^{\circ}$ 1, pp. 15-29. 2004. DOI: 10.1016/j.im.2003.11.002

[30] G. Paré, A.-F. Cameron, P. Poba-Nzaou, and M. Templier. "A systematic assessment of rigor in information systems rankingtype Delphi studies". Inf. Manage. Vol. 50 $\mathrm{N}^{\circ} 5$, pp. 207-217. 2013. DOI: 10.1016/j. im.2013.03.003

[31] M. Keil, H. K. Lee, and T. Deng. "Understanding the most critical skills for managing IT projects: A Delphi study of IT project managers". Inf. Manage. Vol. 50 $\mathrm{N}^{\mathrm{o}} 7$, pp. 398-414. 2013. DOI: 10.1016/j. im.2013.05.005
[32] Y. Wang, I. C. Bilegan, T. G. Crainic, and A. Artiba. "Performance Indicators for Planning Intermodal Barge Transportation Systems". Transp. Res. Procedia. Vol. 3, pp. 621-630. 2014. DOI: $10.1016 /$ j.trpro.2014.10.041

[33] C. Salas, Y. José, C. Pérez, and N. Jaime. "Simulación y optimización para dimensionar la flota de vehículos en operaciones logísticas de abastecimiento-distribución". Ingeniare Rev. Chil. Ing. Vol. $23 \mathrm{~N}^{\circ} 3$, pp. 372-382. 2015. DOI: $10.1080 / 00207543.2012 .709646$

[34] A. Baublys and A. Smičius. "Models of freight transport system development" Transport. Vol. $17 \mathrm{~N}^{\mathrm{o}}$ 6, pp. 205-218. 2002. DOI: $10.1080 / 16483840.2002 .10414045$

[35] R. Ballou, "Logística: administración de la cadena de suministro". Prentice-Hall International. 5th ed. México, México. pp. 789. 2004. ISBN: 70-26-0540-7.

[36] D. Bowersox, D. Closs, and M. Cooper. "Supply Chain Logistics Management". McGraw-Hill. 4th ed. New York, Estados Unidos, pp. 496. 2010. ISBN: 978-0078024054

[37] S. Asadi. "12 - Logistics System: Information and Communication Technology", in Logistics Operations and Management, R. Z. Farahani, S. Rezapour, and L. Kardar, Eds. London: Elsevier, 2011, pp. 221-245. ISBN: 978-0-12-385202-1

[38] C. A. Serna, M. D. Arango and J. A. Gómez. "Dinámica de Sistemas para la Evaluación de Corredores Logísticos". Editorial Bonaventuriana. 1st ed. Medellín, Colombia. 2016, pp. 20-37 ISBN: 978-958-8474-57-1

[39] S. Liu, M. Leat, J. Moizer, P. Megicks, and D. Kasturiratne. "A decision-focused knowledge management framework to support collaborative decision making for lean supply chain management". Int. J. Prod. Res. Vol. $51 \mathrm{~N}^{\circ}$ 7, pp. 2123-2137, 2013. DOI: 10.1080/00207543.2012.709646 